\title{
Upgrade of NSRL Optical Klystron for FEL*
}

\author{
LI Ge, LIU Jin-ying, ZHANG Peng-fei, XU Hong-liang, HE Duohui, WANG Yong, JIA Qi-ka \\ National Synchrotron Radiation Laboratory of USTC, Anhui Hefei 230029, P.R. China
}

\begin{abstract}
A symmetry Optical Klystron (OK) will be upgraded to asymmetry structure for generating more powerful coherent harmonic FEL after measuring buncher parameter from the OK spontaneous emission spectrum. The period of independently adjusted modulator will be extended from $7.2 \mathrm{~cm}$ to $9.2 \mathrm{~cm}$ for higher operation energy that will facilitate the progress of Coherent harmonic generation (CHG) FEL experiments. The upgraded OK parameters are listed and checked by the 3dimensional electromagnetic computation code. Special considerations are given for possible upgrade schemes.
\end{abstract}

\section{INTRODUCTION}

One way of Free Electron Laser (FEL) is coherent harmonic generation by firing the electron beam passing through an optical klystron with an external laser, which induces the energy modulation in the modulator by interaction with the optical field. It is transformed into density modulation (bunching) after passing the buncher, and then, the fundamental and odd harmonic coherent radiation is produced in the radiation undulator $[1,2]$.

For this purpose, one symmetry optical klystron (OK) with identical two undulators on its two sides will be upgraded to asymmetry structure for generating more powerful coherent harmonic after measuring buncher parameter from the $\mathrm{OK}$ spontaneous emission spectrum $[3,4,5]$. The present $12 \times 7.2 \mathrm{~cm}$ period modulator will be upgraded to $10 \times 9.2 \mathrm{~cm}$ by extending $5.6 \mathrm{~cm}$ magnet system on its ends. In this case, higher operation energy will facilitate the match between the $\mathrm{OK}$ and storage ring, which makes experiments easily implemented. The upgraded OK can be operated above the injection energy i.e. $200 \mathrm{MeV}$ for coherent harmonic experiments. The Hefei Light Source (HLS) is routinely ramping from the injection energy of $200 \mathrm{MeV}$ to the operation energy of $800 \mathrm{MeV}$.

The analysis equations for upgrading the $\mathrm{OK}$ are given. The interested induction field is checked by the finite element computation, the 3-dimensional electromagnetic computation software, Opera-3d. The measured B-H curve of the magnet is used in the computation. The typical parameters of the upgraded optical klystron are given. The magnet gaps of the three $\mathrm{OK}$ undulator sections can be independently tuned from $40 \mathrm{~mm}$ to $140 \mathrm{~mm}$. The experimental program to measure the coherent harmonic of the $\mathrm{OK}$ is given.

*Revised by LI Ge, NSRL, June 25, 2001.

"lige@ustc.edu.cn, Projects of Chinese 211 \& high-tech 863-410-8-2.

\section{BASIC THEORY}

\subsection{Emission wavelength from undulator and resonant energy of electron beam}

Emission wavelength from axis of plane undulator is given as ${ }^{[1]}$ :

$$
\lambda=\frac{\lambda_{0}}{2 i \gamma^{2}} \cdot\left(1+\frac{k^{2}}{2}+\gamma^{2} \vartheta^{2}\right), i=1,2,3,
$$

Where, $\vartheta$ is the angle with respect to the beam axis, $i$ is harmonic number, $\gamma$ is Lorentz factor, $\mathrm{k}=0.934(\mathrm{By} / \mathrm{T}) \cdot(\lambda \mathrm{o} / \mathrm{cm})$ is the undulator deflection factor, By is the peak vertical field, given as [6]:

$$
\begin{aligned}
& B_{y}=\frac{2 B_{r} \cdot \sin \frac{\varepsilon \cdot \pi}{M}}{\frac{\pi}{M}} \cdot\left(1-e^{-\frac{2 \pi h}{\lambda_{u}}}\right) \cdot e^{-\frac{\pi g}{\lambda_{u}}} \\
& \stackrel{M=4}{\longrightarrow} B_{y}=1.4261 B_{r} \cdot e^{-\frac{\pi g}{\lambda_{u}}}
\end{aligned}
$$

pure permanent magnet undulator;

Where, $B_{r}$ is Magnet remanence, $M$ is the number of magnet blocks per period on one side, $\lambda$ is period, $h=\lambda / 4$ is the height of blocks, $g$ is magnet gap, $\varepsilon=4 \mathrm{~h} / \lambda$ is the fill factor of undulator.

$$
B_{y}=3.44 e^{-\frac{g}{\lambda_{u}}\left(5.08-1.54 \frac{g}{\lambda_{u}}\right)}
$$

For hybrid undulators [7].

The fundamental resonant energy of electron beam can be given by (1):

$$
E /(M e V)=\frac{\gamma}{1.957}=0.51 \sqrt{\frac{\lambda_{0}}{2 \lambda}\left(1+\frac{k^{2}}{2}\right)}
$$

It's shown from equation (2) that increasing the period length of modulator and decreasing the wavelength of the fired laser could enhance the resonant energy of electron beam.

\subsection{Analysis Computation to Upgrade schemes}

Five experimental Schemes of OK are computed using analysis equation 1-4, which are listed in table1. The remanence conditions $B_{r}=1.2 T$ and $B_{r}=1.25 T$ offered by magnet supplies are respectively used in pure permanent magnet (PPM) modulators of scheme 1 and 3 . 
Table1: Five possible upgraded OK parameters in experimental Schemes

\begin{tabular}{|l|l|l|l|l|l|}
\hline Schemes & $\begin{array}{l}\text { Modulator Period/cm } \\
\text { @ magnet gap }\end{array}$ & $\begin{array}{l}\text { Wavelength of } \\
\text { seed laser/nm }\end{array}$ & Peak field By/T & $\mathrm{K}$ & $\mathrm{E} /(\mathrm{MeV})$ \\
\hline 1. PPM & $7.2 @ 4 \mathrm{~cm}$ & 1060 & 0.2989 & 2 & 163 \\
\hline 2. Hybrid & $7.2 @ 4 \mathrm{~cm}$ & 532 & 0.329 & 2.2 & 246.33 \\
\hline 3. PPM & $9.2 @ 4 \mathrm{~cm}$ & 532 & 0.4368 & 3.75 & 425.3 \\
\hline 4. Hybrid & $9.2 @ 4 \mathrm{~cm}$ & 532 & 0.5 & 4.34 & 484.45 \\
\hline 5. Hybrid & $10 @ 4 \mathrm{~cm}$ & 532 & 0.577 & 5.39 & 616 \\
\hline
\end{tabular}

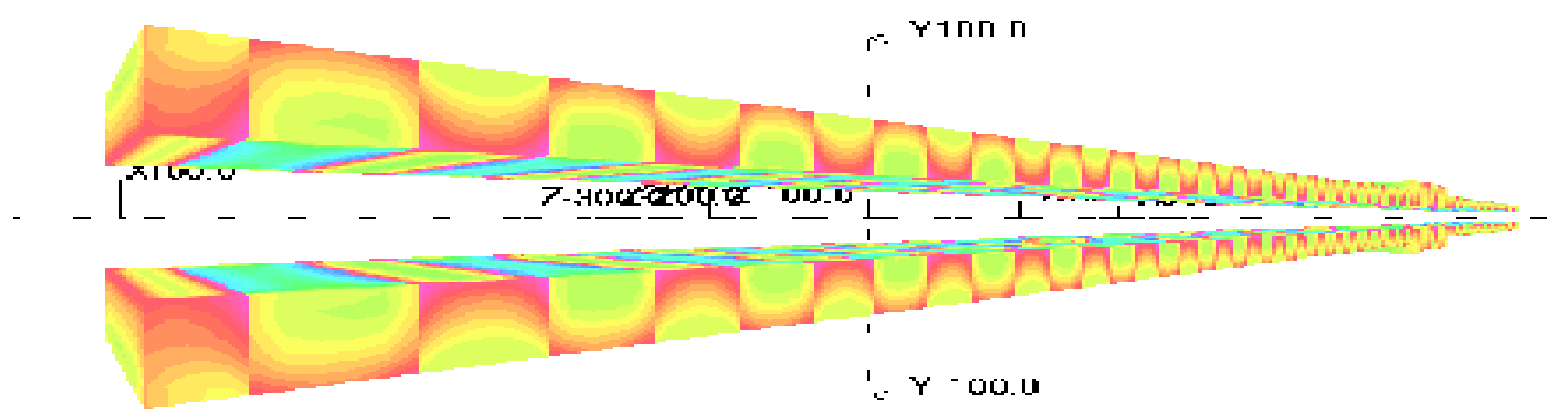

Fig. 1: The induction field of Optical klystron

\subsection{Upgraded Asymmetry OK}

The main parameters of the possible upgrade schemes of optical klystron are listed in table 1 . The scheme 3 is selected for having magnet blocks of $23 \times 23 \times 100 \mathrm{~mm}^{3}$ in hand although No.5 is the best upgrade scheme for the energy of electron beam in this scheme is $616 \mathrm{MeV}$, where the designed HLS $800 \mathrm{MeV}$ storage ring is very stable to be operated with the $\mathrm{OK}$ without matching exploration between them.

\section{$2.43 d$ Finite Element (FE) Computation to the Upgraded Asymmetry OK}

Fig.1 illustrates the induction field of the $3 d$ electromagnetic computation model of the upgraded OK designed by scheme 3 . As shown in fig.2, the measured $\mathrm{B}-\mathrm{H}$ curve of the magnet is used in the computation. The computed vertical field in the beam axis is shown in fig. 3. Typical OK parameters are listed in table 2.

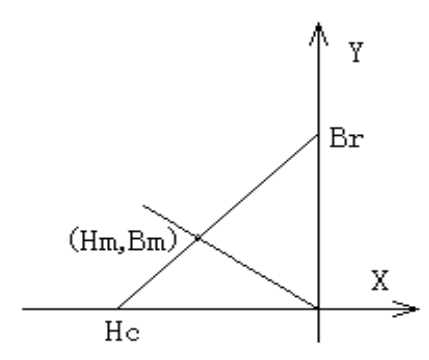

Figure 2. The $B_{r}-H_{c}$ curve of PM Material 
Table 2: The OK typical magnetic parameters computed by $3 \mathrm{~d}$ finite element method and analytic equations

\begin{tabular}{|l|l|l|l|}
\hline & Modulator & Buncher & Radiator \\
\hline Gaps/mm & 36 & 36 & 36 \\
\hline Periods/cm & 9.2 & 21.6 & 7.2 \\
\hline $\begin{array}{l}\text { Computed by } \\
\text { OPERA3d By } \\
/ \mathrm{T}\end{array}$ & 0.506 & 0.758 & 0.3523 \\
\hline $\begin{array}{l}\text { Computed by } \\
\text { analysis } \\
\text { equation By /T }\end{array}$ & 0.5214 & 1.01 & 0.3557 \\
\hline $\begin{array}{l}\text { Errors between } \\
\text { them }\end{array}$ & $3.04 \%$ & $33 \%$ & $1 \%$ \\
\hline period number & 10 & 1 & 12 \\
\hline
\end{tabular}

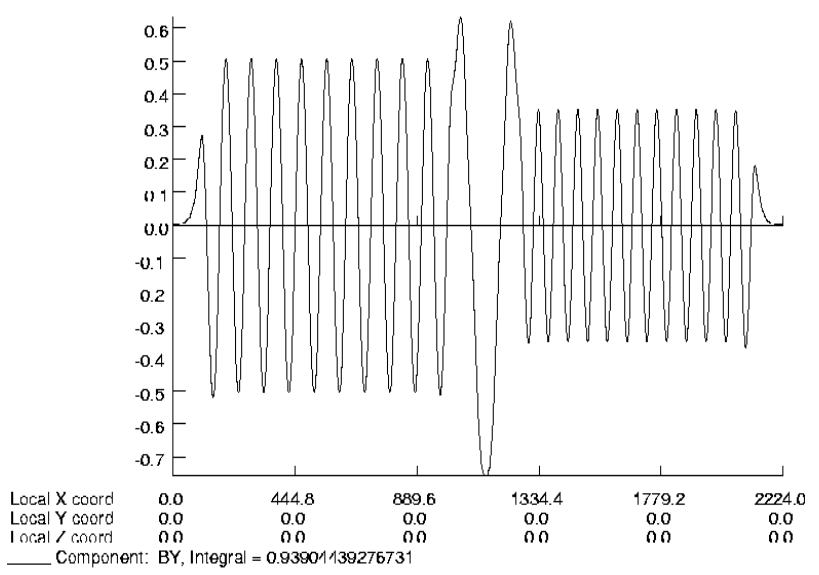

Fig. 3: The computed vertical field in the beam axis

\section{CONCLUSIONS}

3.1 Matching between the OK and the storage ring with electrons beams is the key issue for successful Experiments. Experiments show that HLS storage ring can be normally operated with the present OK above $530 \mathrm{MeV}$ without compensation. So, the No.5 is the best upgrade scheme, which use $10 \mathrm{~cm}$ period hybrid undulator as modulator of CHG FEL experiments for the energy of electron beam in this scheme is $616 \mathrm{MeV}$, where the upgraded OK can be incorporated with HLS storage ring without matching for CHG FEL experiments.
3.2 The present selected scheme 3 still needs matching exploration between HLS storage ring test and the upgraded OK, which use $9.2 \mathrm{~cm}$ period PPM undulator as modulator of CHG FEL experiments for the energy of electron beam in this scheme is $425.3 \mathrm{MeV}$. Even with extremum condition by closing the modulator gap to $36 \mathrm{~mm}$, the fundamental resonant energy of electron beam energy could reach $484.85 \mathrm{MeV}$, which is also below the stable energy of $530 \mathrm{MeV}$ in HLS storage ring with OK.

3.3 As shown in table 2, magnetic computations of 1D analysis equations give larger peak field than that of 3D FE methods, normalized errors is increased when the period of undulator becomes larger at the same width and gaps of upgraded OK undulators. 1D analysis equations miss the factors of width effects, which are increased while extending the period length [6].

\section{REFERENCE}

[1] L.-H. Yu, M. Babzien, I.Ben-Zvi etc. 'High-Gain Harmonic-Generation Free-Electron Laser', Science, Vol. 289, Aug. 11, 2000, PP932-934.

[2] S. V. Milton, E. Gluskin and N. D. Arnold etc. 'Exponential Gain and Saturation of a Self-Amplified Spontaneous Emission Free-Electron Laser', Science, Vol. 292, 15 JUNE 2001, PP2037-2041.

[3] Liu Jin Ying, XU hong-liang, DIAO Chao-zheng etc. 'Investigation on enhancement of Modulation factor of the spectrum from OK spontaneous emission', High Energy Physics and Nuclear Physics, No.1, Vol.24. 2000

[4] Jia Qi-ka. 'Optimisation of optical klystron for CHG FEL,' Nucl.Instr.Meth. A407(1998)246-250.

[5] LI, Ge He Duohui, Jia Qi-ka etc. 'Undulator transformed from optical klystron' High Power Laser and Particle Beams v 8 n 1 Feb 1996. p 7-10.

[6] Poole M W, Bennett R J and Walker R P.A Wiggler Magnet for the UK Free Electron Laser Project, Journal de Physique, Colloque C1,supplement au1,Tome 45, janvier1984.

[7] K. Halbach, 'Status of Undulator developments,' Undulator Magnets for Synchrotron Radiation and Free electron laser. ISBN 9971-50-709-9, World Scientic Publishing Co. Pte. Ltd. 1988. P4. 